\title{
Interpreting Heisenberg Interpreting Quantum States
}

\author{
Simon Friederich \\ friederich@uni-wuppertal.de \\ Universität Wuppertal, Fachbereich C -- Mathematik und Naturwissenschaften, \\ Gaußstr. 20, D-42119 Wuppertal
}

\begin{abstract}
The paper investigates possible readings of the later Heisenberg's remarks on the nature of quantum states. It discusses, in particular, whether Heisenberg should be seen as a proponent of the epistemic conception of states---the view that quantum states are not descriptions of quantum systems but rather reflect the state assigning observers' epistemic relations to these systems. On the one hand, it seems plausible that Heisenberg subscribes to that view, given how he defends the notorious "collapse of the wave function" by relating it to a sudden change in the epistemic situation of the observer registering a measured result. On the other hand, his remarks on quantum probabilities as "potentia" or "objective tendencies" are difficult to reconcile with such a reading. The accounts that are attributed to Heisenberg by the different possible readings considered are subjected to closer scrutiny; at the same time, their respective virtues and problems are discussed.
\end{abstract}

\section{Heisenberg and the epistemic conception of quantum states}

The epistemic conception of quantum states is the idea that quantum states are not descriptions of the properties of quantum systems but rather reflect the state assigning observers' epistemic relations to these systems. Although this view has its early roots in the works of Copenhagen adherents Peierls and, maybe (as discussed in this paper), Heisenberg, it has attracted an increasing amount of attention only in recent years. What makes it attractive is that it promises to provide an elegant dissolution of the notorious paradoxes of measurement and quantum non-locality without relying on any technical notions such as hidden variables, branching worlds or spontaneous collapse that are designed just for the purpose of interpretation. Today, there are several different competing attempts of spelling 
out the epistemic conception of states in detail, its most discussed version probably being Quantum Bayesianism, as developed by Fuchs, Caves, and Schack. ${ }^{1}$

While there has been a considerable amount of work to develop a version of the epistemic account of quantum states that is satisfying from a conceptual point of view, there has been no, or only very little, effort to trace back the historical origins of this idea. The present paper is an attempt to partially fill in this gap by considering the question of whether in the writings of the later Heisenberg one can find an account of quantum states which is an early---perhaps the first---clear-cut exposition of the epistemic conception of states. The question may sound as if it were very easy to answer, but, as the present paper will show, this is not the case. Furthermore, there is clearly no consensus on this matter among those who have proposed interpretations of Heisenberg's philosophical writings: Some take it for granted that Heisenberg is an adherent of the epistemic conception of quantum states, whereas others do not even consider it as a possibility. Marchildon, for instance, when he claims that the epistemic conception of states "goes back at least to Heisenberg", evidently presupposes that Heisenberg is a proponent of it, whereas, for instance, Shimony, ascribes to Heisenberg the "profound and radical" thesis "that the state of a physical object is a collection of potentialities"3---a view which, as will be shown, is in strong tension with the epistemic conception of states, to say the least.

While this lack of unanimity may be surprising at first sight, it becomes understandable if one takes a closer look at the passages in Heisenberg's later writings that are relevant for the understanding of his view of quantum states. As will be discussed in later sections of this paper, the main conclusions one is likely to draw as regards his account of quantum states strongly depend on which of his remarks on the subject matter one regards as central. It is therefore small wonder that interpreters focusing on different aspects of his remarks on quantum states have ascribed to him accounts of the nature of quantum states that are completely ad odds with each other.

The present paper aims at a clarification of these issues by investigating more closely the different readings of Heisenberg's remarks on quantum states that seem possible and coherent.

1 For studies defending or developing versions of the epistemic conception of states and views in a similar spirit, see (Fuchs and Peres, 2000), (Mermin 2003, (Caves et al. 2002a), (Caves et al. 2002b), (Fuchs 2002), (Pitowsky 2003), (Caves et al. 2007), (Spekkens 2007), (Fuchs and Schack 2010), (Friederich 2011).

2 See (Marchildon 2004) p. 1454.

3 See (Shimony 1983) pp. 214-215. 
In order to clarify the meaning of the epistemic conception of quantum states, Section 2 contains a brief exposition and motivation of that view. Section 3 focuses on those of Heisenberg's remarks on quantum states which seem to suggest that, indeed, he is a proponent of the epistemic conception of states. Section 4, in contrast, considers remarks which are difficult to reconcile with such a reading and may prompt one to look for alternatives. Examples of alternative readings are given, and their respective problems and advantages are assessed from both an exegetical and a systematic perspective. Section 5 reconsiders the possibility of interpreting Heisenberg as defending a version of the epistemic conception of states, this time by taking into account the challenge posed by the passages that seem to render such a reading difficult. In Section 6, an entirely different perspective on Heisenberg's remarks on quantum states is presented, based on considerations about the more general aims of philosophical writings proposed by Mara Beller. According to that perspective, Heisenberg is an---as regards philosophical matters---opportunistic thinker, whose remarks on the interpretation of quantum mechanics are mainly motivated by dialectical purposes and do not lead to a coherent foundational perspective. The paper closes with a brief concluding remark in Section 7 without ultimately deciding which of the proposed readings of Heisenberg's remarks on quantum states is correct. Its more modest main aim is to spell out the interpretive options and to improve our understanding of the systematic issues involved.

\section{The epistemic conception of quantum states and the measurement problem}

The epistemic conception of states, as already remarked, is the idea that quantum states are not descriptions of the properties of quantum systems but rather reflect the assigning observers' epistemic relations to these systems. What is involved in adopting this view has been clarified by its proponent Rudolf Peierls, a PhD student of Heisenberg in the late 1920s and an important figure of 20th century physics in his own right. The quantum state, according to Peierls, "represents our knowledge of the system we are trying to describe", and an important consequence of this, as he argues, is that the states assigned by different observers "may differ as the nature and amount of knowledge may differ." This latter aspect provides us with a very useful criterion to decide which views can count as varieties of the epistemic conception of states and which not: If quantum states are supposed to reflect the observer's epistemic relations to the quantum systems states are assigned to, then, since

4 See (Peierls 1991) p. 19. 
different observers may in general know different things about the values of observables of a given system, the states assigned by different observers may legitimately be different, depending on the different observers' differing epistemic conditions.

The epistemic conception of quantum states is incompatible with the idea that there is such a thing as an agent-independent "true" quantum state of a quantum system---a quantum state it "is in"---, for if such a state existed, one would have to assign this state to the system in order to assign correctly, and assigning any other state would be wrong. If indeed the quantum states assigned by different observers having different knowledge of a quantum system are supposed to reflect their epistemic relations to the system, these states may evidently be different. This contrasts with the situation in classical mechanics, where it seems natural to regard the points in phase space as the ontic states classical systems are in. In that context, only that point in phase space correctly describes a classical system which is accurately represents its position and momentum. The status of quantum states, on the epistemic conception of quantum states, is closer to that of probability densities defined over phase space than to points in phase space themselves. Probability densities play a fundamental role in classical statistical mechanics, where which probability density one assigns to some system depends on what information about the values of its macroscopic variables one takes into account. The main difference between probability densities in classical statistical mechanics and quantum states as interpreted by the epistemic conception of states is that there exist extremal probability densities expressing complete information about the ontic state of the system (in form of a Dirac-Delta function over phase space) in the classical case, whereas no quantum state is assumed to correspond to a quantum system's ontic state (inasmuch as such a state is assumed at all ${ }^{5}$ ) in an analogous sense. To sum up, only accounts that reject the notion of an observer-independent true quantum state of a quantum system---a quantum state it is in---can be versions of the epistemic conception of quantum states.

The main motivation for taking the epistemic conception of states seriously is that it neatly dissolves the notorious paradoxes of measurement and non-locality. Heisenberg is naturally read as addressing the measurement problem in his remarks on measurement in quantum mechanics by endorsing its dissolution based on the epistemic conception of states, but he does not comment on issues connected to quantum non-locality. I shall therefore focus on the

5 See the distinction between epistemic accounts of quantum states which are based on hidden variables interpretations and others which are not, discussed at the end of this section. 
measurement problem rather than on quantum non-locality in what follows. ${ }^{6}$

The measurement problem arises from the fact that, if one thinks of quantum states as states quantum systems "are in" and assumes that the time-evolution of these states always follows the Schrödinger equation, measurements rarely have outcomes. ${ }^{7}$ In practice, physicists avoid this difficulty by resorting to von Neumann's notorious projection postulate---the "collapse of the wave function"---which states that the density matrix corresponding to the state of the system after measurement is obtained by projecting the pre-measurement state onto the subspace of eigenstates to the observable measured with eigenvalue corresponding to the measured value of that observable.

The projection postulate, however, is strongly disliked by many researchers in the foundations of quantum mechanics, and many of the most prominent interpretations of the theory attempt to present a coherent picture of the world as described by quantum mechanics that avoids it. According to Laura Ruetsche, for instance, measurement collapse is "a Humean miracle, a violation of the law of nature expressed by the Schrödinger equation" ${ }^{\prime \prime}$, and many others would no doubt agree. Such criticism of the projection postulate, however, is compelling and natural only if one thinks of the state and its time-evolution as descriptions of what actually happens to the quantum system to which the state is assigned. The intuitive motivation for the projection postulate is very different and relates to the need of readjusting the state after measurement in order to make it compatible with what one knows of the values of observables of the system after having registered the measured result. Measurement collapse looks completely natural if one regards it as reflecting a sudden change in the state-assigning observer's epistemic situation, not as a sudden change in the properties of the measured system itself. Consequently, by adopting a version of the epistemic conception of states, saying that the state reflects the assigning agent's epistemic relation to the system the state is assigned to, measurement collapse can be justified in an elegant way, and the measurement problem is avoided.

6 See Section 2 of (Friederich 2011) for a discussion of how the measurement problem and the problem of quantum non-locality are dissolved by the epistemic conception of states and Section 3 of (Fuchs 2002) for more detailed considerations in favour of the epistemic conception of quantum states related to quantum nonlocality, based on Einstein's remarks on entangled states.

7 This is at least what happens if one assumes, as usual, the so-called eigenstate-eigenvalue link which says that for a system in a state $\psi$ an observable $\mathrm{A}$ has a definite value a if and only if $\psi$ is an eigenstate of (the operator corresponding to) A with eigenvalue a.

8 See (Ruetsche 2002) p. 209. 
A natural question in this context is in which sense quantum states might be said to reflect the observers' epistemic relations to the quantum systems. There are two fundamentally different types of possible answers to this question. According to the first type of answer, quantum states encode information about underlying "ontic" states, which are conceived as configurations of fundamental parameters that might as well be called "hidden variables". Rob Spekkens advocates this view and motivates it in an intriguing way by showing that many of the most characteristic features of quantum theories can be reproduced in a toy theory that is based on the epistemic conception of states, supplemented with hidden variables in that sense. ${ }^{9}$ Heisenberg, however, strongly criticizes hidden variables interpretations of different sorts and repeatedly argues for the completeness and definiteness of quantum mechanics ${ }^{10}$, so his position is certainly not an epistemic account of states in terms of hidden variables. To obtain a more plausible candidate for Heisenberg's account of quantum states, the question of in which sense quantum states might be said to reflect the observers' epistemic relations to quantum systems must be answered in a different way, namely, by saying that quantum states reflect the observers' expectations as to what the results of future measurements might be. ${ }^{11}$ Accounts that rely in one way or another on this strategy of spelling out the epistemic conception of states are based on the hope that the notorious paradoxes of measurement and non-locality can be evaded without specifying underlying any "ontic" states at all, simply by adopting a certain perspective on the nature of quantum states. In the next section, we will consider whether this perspective is endorsed in Heisenberg's later writings on the interpretation of quantum mechanics.

\section{Heisenberg and the epistemic conception of quantum states: a straightforward case?}

As already announced in the introductory section, the question of whether Heisenberg should be read as defending the epistemic conception of quantum states is not easy to answer. In analogy to Bohr's remarks on the interpretation of quantum mechanics, Heisenberg's philosophical writings on quantum mechanics have invited strikingly different interpretations

9 See (Spekkens 2007).

10 For his criticism of alternatives to the "Copenhagen interpretation", in particular of hidden variable approaches, see Chapter VIII, "Criticism and Counterproposals to the Copenhagen Interpretation of quantum theory", of (Heisenberg 1958).

11 Chris Fuchs, an important proponent of this approach of spelling out the epistemic conception of states, prefers to talk in a more general way of "experimental interventions into nature" instead of "future measurements", see p. 7 of (Fuchs 2002). 
from different interpreters. At least in part, this is due to the fact that his texts on the interpretation of quantum mechanics are directed not only at physicists (or philosophers having a background in physics), but also at a wider audience of interested laymen. His remarks are intended not only to present an interpretation of quantum mechanics for those who are knowledgeable about the underlying mathematical formalism, but also to provide an overview of elementary features of that formalism for those who know nothing about it at all. Trying to express himself in a manner that is comprehensible also to non-physicists, Heisenberg presents his thoughts in an non-technical and sometimes ambiguous way. As will become clear in what follows, his remarks on the nature of quantum states are a case in point in that they allow for a variety of different, mutually incompatible, readings. The discussion will be based on a rather small number of passages I shall quote, considering various possible readings of these, even though there are certainly many more passages in his later writings that might be relevant with respect to the points at issue. The main reason for proceeding in this way is that the other passages seem to be at least as susceptible to different, mutually incompatible readings as those that I shall discuss. It appears to me more useful to focus thoroughly on a rather narrow class of statements which are directly relevant to the present topic than on a larger number of diverse remarks which allow for at least as many different interpretations as well.

The main reason for ascribing to Heisenberg an epistemic account of quantum states is that, when he writes that "[s]ince through the observation our knowledge of the system has changed discontinuously, its mathematical representation also has undergone the discontinuous change and we speak of a 'quantum jump"'12, he relates measurement collapse to a sudden change in the epistemic situation of the observer. Since such an account of measurement collapse seems to presuppose the epistemic conception of states, the quote just given provides evidence that Heisenberg subscribes to that view. The way how he introduces the notion of a quantum state ("probability function") to his readers can be taken to confirm such a reading: ${ }^{13}$

The probability function represents a mixture of two different things, partly a fact and partly our knowledge of that fact. It represents a fact insofar as it assigns at the initial time the probability unity (i.e. complete certainty) to the initial situation: the 
electron moving with the observed velocity at the observed position; "observed" means observed with the accuracy of the experiment. It represents our knowledge insofar as another observer could perhaps know the position of the electron more accurately. The error in the experiment does---at least to some extent---not represent a property of the electron but a deficiency in our knowledge of the electron. Also this deficiency of knowledge is expressed in the probability function. (See (Heisenberg 1958) p. 19.)

Quantum states, according to this statement, depend on the accuracy of the state assigning observers' knowledge of the systems states are assigned to, and in that sense they have an important subjective element. At the same time, they also have an objective element---"a fact", as Heisenberg writes---that manifests itself in the assignment of probability one to the values of observables that one knows after having measured them. The fact that Heisenberg acknowledges such an objective element of quantum states does not by itself pose any problem for the reading of him as endorsing a version of the epistemic conception of states. A natural way of interpreting his claim that the quantum state "represents a fact insofar as it assigns at the initial time the probability unity (i.e. complete certainty) to the initial situation" is to read it as claiming that the values of observables are objective facts about quantum system and that an observer's assignment of a quantum state to a quantum system must be compatible with knowledge about such facts in that the state assigned must ascribe probability one to the values of observables that the observer knows to obtain. If, for instance, an observer knows that the z-component of the spin of a certain electron has the value $1 / 2$, the state he assigns to the system must be an eigenstate of the operator $S_{Z}$ with eigenvalue $1 / 2$. That this state assignment must be in accordance with the observer's knowledge of the value of the spin observable is quite naturally regarded as an "objective element" of the state assigned. This does not contradict the epistemic conception of states as long as one does not make the further claim that the state assigned has to be identified with the true state of the system---the one it "is in". According to this reading of the passage quoted above, the values of observables are objective features of physical reality, whereas the probabilities ascribed to possible measurement outcomes have at least some subjective aspect in that the states from which they are derived are different for observers having different epistemic relations to the system. $^{14}$ than subjective character. Healey's pragmatist interpretation of quantum theory (Healey 2012) for instance, 
Heisenberg claims explicitly that different observers may legitimately assign different states to the same system, namely, with respect to cases where an observer has knowledge of the values of observables of a system (an electron, in his example) which is objectively inferior to the knowledge of another observer who "know[s] the position of the electron more accurately." In the type of situation considered here, an observer who is less well informed about the value of an observable of the system than another may assign a different state than the latter without necessarily making any sort of mistake. Heisenberg does not seem to consider the state assignment of the observer who is less well informed as somehow "wrong" or "incorrect", even though the observer who knows more about the values of observables is certainly more likely to make successful predictions about the results of future measurements of the system.

Let us now consider a slightly different type of situation where different observers have different knowledge of the values of observables of a quantum system in such a way that none of them knows strictly more about the values of observables than the others. What can we say about Heisenberg's position with regard to this more general case? Assuming that he holds that two observers may legitimately assign two different states if one of them knows strictly less about the values of its observables than the other, we can safely conclude that he would hold the same with respect to the type of situation where none of them knows strictly less (or more) than the other. Together with the observation that, in the passage quoted above, he does not speak about the "true" quantum state of a system (or its "true" probability function) these considerations can be taken to suggest that Heisenberg does indeed endorse a version of the epistemic conception of quantum states.

In the following section, I shall turn to other passages in his writings that bear on the interpretation of quantum states, which, unlike the ones considered so far, pose considerable difficulties for interpreting him as endorsing an epistemic account of quantum states.

\section{Probabilities as objective tendencies: the crux with the "subjective element"}

which is at least very close to the epistemic conception in spirit, conceives of probabilities as objective, and similar comments apply to the epistemic account of states developed in (Friederich 2011), where, however, the status of quantum probabilities is not considered explicitly. 
The most important difficulty for reading Heisenberg as defending a version of the epistemic conception of states is that he holds that quantum states contain "statements about possibilities or better tendencies ..., and these statements are objective." Here is a context in which he advances this claim: ${ }^{15}$

The probability function combines objective and subjective elements. It contains statements about possibilities or better tendencies ("potentia" in Aristotelian philosophy), and these statements are completely objective, they do not depend on any observer; and it contains statements about our knowledge of the system, which of course are subjective in so far as they may be different for different observers. In ideal cases the subjective element in the probability function may be practically negligible as compared with the objective one. The physicists then speak of a "pure case.” (See (Heisenberg 1958), p. 27)

By "statements about possibilities or better tendencies" that are contained in quantum states Heisenberg seems to mean statements about the probabilities of the values of observables that are derived from quantum states via the Born rule. When he argues that these statements are "completely objective" and that they "do not dependent on any observer", he seems to endorse an account of quantum probabilities as objective probabilities that is very much in spirit with what is nowadays called the "propensity interpretation" of probability. ${ }^{16}$ Accounts of probability that are based on this interpretation conceive of probability as measuring an object's objective tendency---or disposition---to display a certain property. Propensity views of probability conceive of probabilities as objective in a very strong sense in that they conceive them as (objective) properties of the objects (or events) to which they are ascribed. At the opposite spectrum of accounts of probability are subjectivist ("Bayesian") accounts. These interpret probabilities as degrees of belief of the agents assigning probabilities, for which questions of truth or correctness as regards probability assignments do not make sense. Whereas the propensity view regards probabilities as agent-independent features of the things themselves, probabilities as degrees of belief may differ from agent to agent, without any of them being in error or making any mistake. In order to be compatible with the epistemic conception of quantum states, accounts of quantum probabilities must make at least some

15 See, for instance, (Heisenberg 1984b) p. 240 and (Heisenberg 1958) p. 145-155 for similar remarks on his "Aristotelian" notion of "potentia". (Schiemann 2008) p. 57 gives an argument of why Heisenberg's notion of "potentia" is quite far from the original concept in Aristotle's works.

16 For an illuminating classification of propensity interpretations of probability which also serves as an introduction to the subject see (Gillies 2000). 
small concession to a subjectivist interpretation of quantum probabilities as it has to allow that quantum probabilities may differ for different observers.

Heisenberg's claims on quantum probabilities as "objective tendencies" have indeed been interpreted as evidence that he subscribes to a propensity view of quantum probabilities. Henry P. Stapp, for instance, claims that "[Heisenberg] propos[es], in effect, that certain probabilities defined by the theory be interpreted as the 'objective tendencies', or propensities, for corresponding actual events to occur." ${ }^{, 17}$ According to Stapp, the idea of interpreting quantum probabilities as propensities is a metaphysically significant step in that it "carries quantum theory far beyond the ontologically neutral stance of the strictly orthodox interpretation." ${ }^{18}$ In a similar vein, Abner Shimony attributes to Heisenberg a "profound and radical thesis", namely, "that the state of a physical object is a collection of potentialities." 19 According to these interpretations of Heisenberg's remarks on quantum probabilities his views are a "foray into metaphysics" 20 , as Kristian Camilleri notes, which goes far beyond the mere attempt of clarifying the notions of state and probability as they are used in quantum mechanics.

However, an immediate objection to reading Heisenberg as defending a propensity interpretation of quantum probabilities is that it seems to be incompatible with his claim that measurement collapse reflects the fact that "our knowledge of the system has changed discontinuously." This account of measurement collapse, as already discussed, presupposes that the state subjected to collapse reflects the epistemic situation of the observer and is therefore at odds with the idea that the state describes the system's propensities of displaying certain values of observables. As we have seen in Section 2, the main motivation for adopting the epistemic conception of states is that it permits to interpret measurement collapse in an organic way as reflecting a sudden change in an observer's knowledge of the system.

Those who read Heisenberg as endorsing a propensity view of quantum probabilities may respond to this challenge by pointing to the fact that he classifies as objective only those probabilities assigned to quantum systems in what he calls "pure cases". It is only in these "pure cases" that the "subjective element" of quantum states discussed in the previous section,

17 See (Stapp 2004) p. 42, the emphasis is due Stappp.

18 See (Stapp 2004) p. 42

19 See (Shimony 1983) pp. 214-215.

20 See (Camilleri 2009) p. 153. 
as he claims, becomes "practically negligible". A "pure case", as he uses this expression, is present whenever a system can be considered in complete isolation from its environment, at least to a high degree of precision, so that it need not be regarded as part of a larger, composite system, together with others. In this situation, as Heisenberg claims, it can be described by a pure quantum state (a Hilbert space vector ${ }^{21}$ ), so that we may think of "pure cases" as those where an observer assigns a pure state to a system whose interactions with the environment are negligibly small. Since, according to Heisenberg, the "subjective element" of quantum states is absent in cases that meet this condition, it follows that he regards this element as restricted to situations where a system is not in isolation from its environment, which means that it cannot be well described by means of a pure quantum state. ${ }^{22}$

The idea that pure states, but not mixed states, correspond to the objective properties of quantum systems is familiar from the well-known "ignorance interpretation of mixed states", and it is tempting to read the passage quoted at the beginning of this section as endorsing that view. The ignorance interpretation of mixed states is the view that each quantum state is described by exactly one pure quantum state, and that mixed states should be assigned only by those observers who do not know the exact pure state of the system they are assigning a quantum state to. Thus, on the ignorance interpretation of mixed states a mixed state reflects the assigning observer's ignorance of the actual pure quantum state the system is in. These pure states, in contrast to the mixed states, describe the objective probabilities of quantum systems to display certain properties at a given time. In view of the fact that Heisenberg characterises quantum probabilities as "objective tendencies", arguing that the "subjective element" of quantum states is absent in "pure cases", one might think that the position he defends is a version of the ignorance interpretation of mixed states. However, as will become clear in a minute, this can hardly be the case.

To see why, we have to recall the distinction between "properly" and "improperly" mixed states, originally introduced by d'Espagnat. ${ }^{23}$ The first of these two, the "proper mixtures", are mixed states that are assigned to quantum systems in cases where the state preparation procedure does not narrow down possible states to assign to a unique pure state. It is only in

21 See (Heisenberg 1955) p. 27, for the German original see (Heisenberg 1984a) p. 447. 22 Pure states contrast with mixed states which, in ordinary Hilbert space quantum mechanics, are states that cannot be written in the form of Hilbert space vectors $\psi$, but only as density matrices $\rho$. For density matrices $\rho$ 
these cases that the ignorance interpretation of mixed state makes sense in that it claims that the assignment of a mixed state reflects the observer's ignorance about the pure state the system really is in. In contrast to mixed states of this type, there are also the so-called "improper mixtures" to which the ignorance interpretation of mixed states cannot be applied. "Improper mixtures" are mixed states which one obtains for systems that are subsystems of larger many-component systems by performing the trace operation over the degrees of freedom of the other subsystems. If we denote by $\rho$ the density matrix assigned to the combined (many-component) system and label the subsystems by the index i, the reduced state of the subsystem $j$ is given by $\rho_{j}=\operatorname{Tr}_{i \neq j}(\rho)$, where the trace $\operatorname{Tr}_{i \neq j}$ is taken over the degrees of freedom of the other subsystems $i \neq j$. In the generic case, the state $\rho$ assigned to the combined system cannot be written as a (tensor) product state of the reduced density matrices $\rho_{i}$ of its subsystems. This is equivalent to saying that the state $\rho$ will normally be an entangled state and that the reduced states $\rho_{\mathrm{i}}$ will have the form of mixed states, which means that for them the inequality $\operatorname{Tr}\left(\rho_{\mathrm{i}}{ }^{2}\right)<1$ holds. However, mixed states that have been obtained in this way as reduced density matrices via performing the trace cannot be given an ignorance interpretation in the sense of reflecting ignorance about the "true" pure states the subsystems $i$ are in. The reason for this is that the state $\rho$ assigned to the combined system in general does not have the form of a mixture of states which are products of pure states for the subsystems $\mathrm{i}$ in such a way that the reduced density matrices $\rho_{i}$ are mixtures of these pure states with the coefficients used in the decomposition of the state $\rho$ of the combined system. Consequently, the reduced density matrices $\rho_{i}$ cannot be given an interpretation as reflecting ignorance about any pure states the subsystems $i$ are in, so the ignorance interpretation of mixed states applies only to proper, not to improper, mixtures.

The ignorance interpretation of mixed states that conceives of the assignment of mixed states as reflecting ignorance about the true pure states quantum systems are in, as we see, applies only to "properly" mixed states, not to "improper mixtures". Heisenberg's remarks on mixed states as expressing some sort of "ignorance", however, do not refer to systems where the state preparation procedure does not narrow down possible states to assign to a unique pure state, but to systems that are not in isolation from their environment and must therefore be considered as subsystems of a larger system. This concerns, in particular, quantum systems 
that are measured by means of a measuring apparatus with which they interact. Concerning these systems Heisenberg claims that "it is very important to realize that our object [being measured] has to be in contact with the other part of the world, namely, the experimental arrangement, the measuring rod, etc., before or at least in the moment of observation." ${ }^{24}$ According to the considerations presented before, the only state one can assign to the system being measured when it is coupled in this way to the measuring apparatus is a mixed stated given as a reduced density matrix. Heisenberg explicitly ascribes a "subjective element of incomplete knowledge" to this state when he writes that "[a]fter this [measurement] interaction has taken place, the probability function contains the objective element of tendency and the subjective element of incomplete knowledge, even if it has been a 'pure case' before." ${ }^{25}$ This "incomplete knowledge", however, as we have seen, cannot be incomplete knowledge about the true pure state the measured system is in, so, we might ask, what kind of incomplete knowledge can it be? Heisenberg's answer to this question is that it is incomplete knowledge about the microscopic details of the rest of the world to which the measured system, via the measurement device, must be coupled. The "subjective element" which, according to himself, is present in (improperly) mixed states relates to an ignorance on behalf of the observer in that it reflects her "uncertainties of the microscopic structure of the whole world." ${ }^{26}$ This statement makes it clear that Heisenberg is not endorsing the conventional ignorance interpretation of properly mixed states in terms of ignorance about underlying pure states, but another, more idiosyncratic, ignorance interpretation of improperly mixed states, holding that these reflect ignorance of an altogether different sort, namely, ignorance of the microscopic details of the rest of the world. Is this a reasonable type of an "ignorance interpretation" of mixed states?

There are several aspects to this question. First, we should note that Heisenberg has indeed good reasons not to endorse the conventional ignorance interpretation of mixed states as reflecting incomplete knowledge of the true pure state the system is in, for this interpretation, as is widely known, runs straight into the measurement problem. The conventional ignorance interpretation of mixed states would have a chance of solving the measurement problem only in case the post-measurement state of the measured system---or that of the measured system together with the measuring apparatus---would be a mixed state in form of a proper mixture of pure states associated one-to-one to the possible outcomes of measurement. However, this is

24 See (Heisenberg 1958) p. 27, see also (Heisenberg 1955) p. 27.

25 See (Heisenberg 1958) p. 28. The same point is made in (Heisenberg 1955) p. 27-28.

26 See (Heisenberg 1958) p. 27. 
not the case, for the state of the measured system (or that of the measured system together with the apparatus, if the environment is included) is an improper mixture for which, as we have seen, no ignorance interpretation in terms of underlying pure states can be given. Consequently, Heisenberg's strategy to characterise improper, rather than proper, mixtures as reflecting incomplete knowledge is in a sense well-motivated.

The problem with his supposed strategy to spell out ignorance in terms of "uncertainties of the microscopic structure of the whole world", however, is that it does not allow for an elegant dissolution of the measurement problem either. If the states of the systems being measured always were proper mixtures, the interpretation of mixed states in terms of ignorance about underlying pure states could justify measurement collapse by relating it to a change in knowledge about the underlying pure state. As we have seen, however, the state of the measured system ${ }^{27}$, taken by itself, must be an improper, rather than a proper, mixture, so that it cannot be interpreted as reflecting ignorance of that sort. Heisenberg's idea that mixed states may reflect ignorance of an altogether different sort, namely, ignorance about "uncertainties of the microscopic structure of the whole world" does not help us solving the measurement problem in an analogous fashion. A measurement normally does not provide us with new information about the "microscopic structure" of the rest of the world, and even if it did, it remains unclear why this information should necessitate a sudden change of state for the system being measured in exactly the way prescribed by von Neumann's projection postulate. Heisenberg's defence of measurement collapse was that "through the observation our knowledge of the system has changed discontinuously." However, it is dubious why a change in our "uncertainties of the microscopic structure" of the rest of the world should affect the state of the system being measured and force it to undergo a "discontinuous change".

As we have seen before, the problem with ascribing to Heisenberg a propensity view of quantum probabilities is that such a view threatens to be incompatible with his defence of measurement collapse as reflecting a sudden change in the knowledge of the observer. To avoid this problem, we considered Heisenberg's claim that mixed quantum states---improper mixtures, as I have argued---have an epistemic aspect in that they reflect ignorance in form of "uncertainties of the microscopic structure of the world." The result we have obtained is discouraging, for it remains completely unclear in which sense uncertainties of that sort

27 Referring to "the" state of the measured system I presuppose an ontic, rather than an epistemic account of states. This is justified in the context of the present section, where I focus on readings of Heisenberg as endorsing an ontic account of states. 
should be relevant for a sudden change of the state of a quantum system being measured. The most optimistic conclusion that can possibly be drawn at this stage is that the problem of interpretation we encounter here is just due to the fact that Heisenberg does not spell out his ideas in greater detail. However, we should also consider alternative readings.

One possible reaction to the problem of combining Heisenberg's remarks on quantum probabilities as objective tendencies with his account of measurement collapse is to simply ignore the remarks on collapse, for instance by interpreting them as mere rhetorical moves to make the apparent abruptness and unruliness of collapse seem more acceptable. ${ }^{28}$ An interpretive reading along these lines is developed by Henry P. Stapp, who claims that according to Heisenberg collapse is a physical process that from time to time interrupts unitary time-evolution as governed by the Schrödinger equation in an irregular manner. According to this supposed view of Heisenberg, "[t]he fundamental dynamical process of nature is no longer one single uniform process, as it is in classical physics. It consists rather of two different processes." ${ }^{29}$ The smooth, unitary time-evolution and the discontinuous statechange of collapse are both regarded as physical processes, and the features of reality that correspond to the sudden state-changes of collapse are certain randomly occurring "actual events". Quantum mechanical probabilities are objective in this view in that they measure observer-independent tendencies of the corresponding actual events to occur. The picture of reality that emerges from this interpretation exhibits certain similarities to spontaneous localisation theories such as the model developed by Ghirardi, Rimini, and Weber ("GRW"), which modifies standard quantum mechanics by complementing time-evolution according to the Schrödinger equation with randomly interspersed spontaneous localisation processes. In comparison to GRW-theory, Heisenberg's position as construed by Stapp has the disadvantage that it does not give a quantitative account of the frequency and dynamics of how these processes of spontaneous localisation occur.

Stapp's interpretation of Heisenberg's remarks on quantum probabilities, as we see, is unsatisfying for systematic reasons, but, more importantly, it is unconvincing as a reading of Heisenberg on exegetical grounds. It identifies the transition from the realm of "potentiality" to that of "actuality" in form of an "actual event" with the discontinuous change of state in measurement collapse, whereas Heisenberg categorically claims that "the transition from the

28 For a reading that adopts this idea and develops it further, see Section 6 of this paper.

29 See (Stapp 2004) p. 41. 
'possible' to the 'actual'",30 has nothing to do with that change, which results from the "discontinuous change of our knowledge in the instant of registration." 31 According to Heisenberg, the transition from the possible to the actual coincides not with measurement collapse, but rather with the "physical act of observation", namely, the "interaction of the object with the measuring device" ${ }^{\prime 2}$, which, as he suggests, must be sharply distinguished from collapse. Stapp's account, aside from ignoring Heisenberg's claim on collapse as reflecting a sudden change of knowledge of the observer, thus fails for both systematic and exegetical reasons. In the next section, I consider a completely different reading of Heisenberg's remarks of quantum states that takes his account of the "the transition from the 'possible' to the 'actual"' just quoted as its starting point and reconsiders the idea that he should be read as endorsing a version of the epistemic conception of states.

\section{The epistemic conception reconsidered}

Kristian Camilleri has recently proposed a novel interpretation of Heisenberg's later writings according to which the dichotomy between the "actual" and the "possible" should be conceived as a contrast between two different forms of description rather than a contrast between two different metaphysical categories. According to this reading, "the transition from the 'possible' to the 'actual"' should not be construed as a physical process occurring in nature, but rather as a change in the language we use at different stages in our description of the measurement process. In particular, as Camilleri contends, this transition has nothing to do with measurement collapse :

The 'transition from the potential to the actual' is therefore completely misunderstood if it is interpreted as a collapse of a physically real extended wave-packet in space. Nor should it be interpreted in Berkeley's sense: esse est percipi. Rather, we must understand the 'actual' and the 'possible' as two modes of description, both of which employ the language of time and space at some level. The transition from potentiality (a quantum-mechanical description) to actuality (a classical space-time description) must be understood as a transition from one mode of description to another. The two modes of description---the possible and the actual---are deemed complementary in Heisenberg's, though not in Bohr's, sense of the term. (See

30 See (Heisenberg 1958) p. 28, see also (Heisenberg 1955) p. 28.

31 See (Heisenberg 1958) p. 29.

32 See (Heisenberg 1958) pp. $28 \mathrm{f}$. 
(Camilleri 2009) p. 170.)

Camilleri backs up his interpretation by means of a large number of passages in Heisenberg's later writings on the topic of language which he reads as endorsing an account which, according to Camilleri, "could be termed a 'quasi-transcendental conception of language'",33 Without going into the details of Camilleri's reading of Heisenberg on the role of language in quantum mechanics, I take it as a starting point for an interpretation of Heisenberg's remarks on quantum states as containing statements about "objective tendencies" that avoids the propensity interpretation of probabilities and fits well with the epistemic conception of states.

Let us start from Camilleri's reading of Heisenberg's remarks that interprets the distinction between actuality and potentiality corresponds as a distinction between two modes of description, namely, a "classical space-time description" and a "quantum-mechanical description". Does this distinction go well with the epistemic conception of states that regards quantum states as reflecting the state assigning agents' epistemic conditions? I shall argue that it does, at least if the epistemic conception of states is spelled out in a certain way, namely, as saying that quantum states reflect the observers' knowledge of the values of observables. ${ }^{34}$ If the epistemic conception of states is spelled out in that way, one might indeed somewhat schematically describe it as recognising fundamentally different "actuality" and "potentiality" modes of description in quantum mechanical practice.

The "actuality mode" is exemplified by sentences which report an observer's knowledge of the values of observables such as in the form "The value of the observable A of the system $\mathrm{s}$ lies in the set $\Delta$." Sentences of that form are referred to as "non-quantum magnitude claims" (NQMCs) by Richard Healey ${ }^{35}$, and I adopt this terminology in what follows. In accordance with Camilleri's characterisation of the "actuality mode of description" NQMCs can be regarded as "classical space-time descriptions" in that they describe, up to a certain accuracy, the properties of a physical system by means of the vocabulary of classical physics, without employing any probabilistic notions. ${ }^{36}$ The "potentiality mode of description", on the other

33 See (Camilleri 2009) p. 152. Heisenberg expresses his views of the overarching importance of language, for instance, when he writes: "Language is, as it were, a net spread between people, a net in which our thoughts and knowledge are inextricably enmeshed." (See (Heisenberg 1971), p. 138.) See also Chapter X of (Heisenberg 1958) on "Language and Reality in Modern Physics".

34 For a version of the epistemic conception of states based on this idea, see (Friederich forthcoming). 35 See (Healey 2012), Section 3.

36 This characterisation remains a little unfortunate, however, because knowledge of the values of non-classical observables such as spin can be formulated in an exactly analogous manner. 
hand, is given by the assignment of quantum states to quantum systems, together with the derivation of probabilities for the values of observables from the states via the Born Rule. This is certainly not an "actuality mode of description" from the perspective of the epistemic conception of states, which denies that assignments of quantum states do ever report any facts about quantum systems. From the perspective of the epistemic conception of states, quantum states are not really descriptions of quantum systems at all, at least not in a more narrow sense of "description", where two descriptions of the same object cannot both be correct if they are mutually incompatible the sense in which assignments of different probabilities to one and the same value of a certain observable are. However, one may want to call the assignment of quantum states to quantum systems a "mode of description" in a wider sense of the word "description", and in that case one will certainly have to call it a "potentiality mode" rather than an "actuality mode" in that it uses probabilistic notions and, as already remarked, does not describe any "actual" facts pertaining to the objects.

From the perspective of such an epistemic account of states, the transition from "potentiality" to "actuality" amounts to a switch in ones "mode of description" of a system treated quantum mechanically. Assume that you have assigned a quantum state to a system ("potentiality mode"), thereby making some predictions as to what, for instance, the results of future measurements will be, and that, subsequently, on the basis of incoming measurement data, you are able to formulate an "NQMC", that is, a statement about the values of observables measured ("actuality mode"). The new information of the values of observables obtained makes it necessary to adjust the state assigned to the system by means of the discontinuous change that is measurement collapse, but this change, in contrast to the one described before, occurs on the level of time-evolution of quantum states alone. In other words, it appears completely within the sphere of the "potentiality mode of description", in agreement with Heisenberg's claim that collapse has nothing to do with the transition from potentiality to actuality but merely signals a change in the representation of our knowledge.

Some adherents of the epistemic conception of states may not like to characterise the different uses of language in quantum mechanics in terms of the notions of "potentiality" and "actuality", for these are metaphysically charged notions that may cause serious misunderstanding. Nevertheless, as we have seen, versions of the epistemic conception of states that regard quantum states as reflecting the observer's epistemic situation in the form of reflecting knowledge of the values of observables seem to go well with Camilleri's 
interpretation of Heisenberg's distinction between the 'actual' and the 'potential' as two contrasting modes of speech. What remains to be shown is that it is compatible with Heisenberg's claim that the probabilities derived in "pure cases" are "completely objective" and that the "subjective element" can be neglected in situations where pure states are assigned. This claim is in tension with the epistemic conception of states according to which different observers having different knowledge of the same quantum system may always assign different states to it. This "subjective element" of observer dependence characterises all quantum states, both pure and mixed, according to the epistemic conception of states.

The question of how to address the status of pure states is a very general challenge for the epistemic conception of states that arises not only in connection with the interpretation of Heisenberg's remarks on quantum states. It can be formulated as follows: Whenever an observer has knowledge of the values of observables of a quantum system that narrows down possible states to assign to a unique pure state, this knowledge about the values of observables cannot be improved any further due to the uncertainty principle. Take the case of an observer who measures, say, the z-direction $\mathrm{S}_{\mathrm{Z}}$ of electron spin by means of a Stern-Gerlach device. ${ }^{37}$ Having observed an electron leaving the device on an "up"-trajectory, she knows that the value of $S_{Z}$ is $1 / 2$. Only the assignment of the state $\mid{ }_{z}>$, the eigenstate of $S_{Z}$ to the eigenvalue $1 / 2$, is compatible with that piece of knowledge. If she proceeds to measure another direction of spin, say $\mathrm{S}_{\mathrm{X}}$, her knowledge of the value of $\mathrm{S}_{\mathrm{Z}}$ will get lost according to the uncertainty principle. Her knowledge of the values of observables, in this case spin in various directions, may be changed due to measurement, but it cannot be improved in that for every bit of information she obtains of the value of spin in a certain direction she loses information of the value of spin in another direction. Any assignment of state that is made on the basis of knowledge which is not improvable in that sense can be seen as objectively privileged as compared to all other assignments that observers may make on the basis of different knowledge of the values of observables. It is natural to conclude from this that a state assignment which is "privileged" in that sense is an assignment of the true quantum state of the system---the one it "is in". This conclusion is incompatible with the epistemic conception of states, and the line of thought just presented therefore provides a general challenge for the epistemic conception of states that is relevant not only for the question of how to interpret

37 I focus on the spin degree of freedom, where the associated Hilbert space has only (complex) dimension 2, to make the argument as perspicuous as possible. Completely analogous considerations apply for the orbital degrees of freedom of whatever particle. 
Heisenberg's remarks.

Fortunately---from the perspective of the epistemic conception of states---the challenge just formulated can be answered. To see how, one should first note that the epistemic conception of states can allow that a state that is assigned by an observer whose knowledge of the values of observables cannot by improved any further has a special and privileged status. This, however, does not necessarily mean that this state can count as the true state of the system in the sense of a state the system is in, for one need not conclude that this state corresponds to a property which the system has independently of someone being there who happens to assign it. The proponent of the epistemic conception of states may well regard the expression "state assigned by those whose knowledge about the values of observables cannot be further improved" as having a referent only if an agent happens to be there who really has such excellent knowledge. Sometimes there might indeed be agents having knowledge of the values of observables which narrows down possible states to assign to a uniquely determined pure state, but the assumption that even when there are no agents having such knowledge, there exists some state which would have to be assigned by anyone assigning a state to the system is at odds with the principles of the epistemic conception of states itself.

Consequently, even if one concedes that from time to time agents may have knowledge of the values of observables which cannot be further improved, this does not mean that one is also committed to the view that for any system there exists some state it is in.

This line of thought is not found in Heisenberg's writings, but we can speculate that he might have accepted it, to combine an epistemic account of quantum states with the view that the probabilities derived from quantum states in what he calls "pure cases" are objective. Heisenberg's habit of calling these probabilities "objective tendencies" is misleading on this reading, for this wording invites an interpretation of him as endorsing a propensity view of quantum probabilities with which the epistemic conception of states is incompatible. From the perspective of the epistemic conception of states, the probabilities derived from pure states are not objective simpliciter, but, nevertheless, objectively privileged over any other probabilities that different observers might have assigned on the basis of poorer knowledge of the values of observables. This can be seen as a sense in which, in accordance with Heisenberg's claims, the probabilities ascribed to the values of observables in "pure cases", that is, the probabilities derived from pure states, can be regarded as "objective" to a higher degree than others without obtaining any conflict with the epistemic conception of states. 
On the one hand, the reading of Heisenberg according to which he holds that the probabilities derived from pure states are "objective" in the (weak) sense of "objectively privileged" just specified may seem not as natural as the reading discussed in the previous section according to which he defends a propensity view of quantum probabilities. On the other hand, it has the virtue of being compatible with the epistemic conception of states and, therefore, with his defence of measurement collapse as deriving from the instantaneous change in the knowledge of the observer when registering the measured result. The two readings developed in this and the previous section are very different, and even if my sympathies are slightly in favour of the reading proposed in this section, I still find it difficult to say which of them is more likely correct. In the following section, the last of this paper, I present yet another reading of Heisenberg's remarks on quantum states, based on the idea that there might just be no coherent account of the nature of quantum states and probabilities in his later writings at all.

\section{Heisenberg as a philosophical opportunist?}

As we have seen in the previous sections while discussing possible readings of Heisenberg's remarks on the nature of quantum states, the interpretation of these remarks is an intricate task. In this section, I consider as a possible reaction to the interpretive problems we have encountered the idea that finding a coherent account of quantum states in Heisenberg's later writings is so difficult simply because there is no such account to be found at all.

A reading that incorporates this idea might be based on a general approach to the interpretation of Heisenberg's philosophical remarks on quantum mechanics developed by Mara Beller. Beller sees Heisenberg as chiefly concerned with establishing and defending his own reputation as a physicist whose discoveries are of uttermost relevance not only for physics itself, but also for age-old debates outside physics in philosophy. She claims that Heisenberg "wanted to be the new Kant" 38 in that he intended to make a lasting contribution to the debate about causality that goes back to Hume and Kant by arguing "that 'there seems to be the strongest evidence' for the 'final' renunciation of causality and objectivity." 39 Despite his strong claims on the irrevocable "inevitability of acausality" Heisenberg's claims by 
means of which he intends to support his philosophical conclusions are, according to Beller, "built on shaky circular arguments, on intuitively appealing but incorrect statements, [and] on metaphorical allusions. ${ }^{940}$ Beller holds that philosophical ideas such as that of the indispensability of classical concepts are endorsed by Heisenberg only in a "local and opportunistic fashion"41, that is, they are used for rhetorical purposes only, not as wellweighed contributions to substantial philosophical debates. According to Beller, Heisenberg's celebrated use of the positivist "principle of elimination of unobservables" 42 in his seminal 1925 paper "Quantum-theoretical re-interpretation of kinematic and mechanical relations"43 can be regarded as an early case in point, since, as she concludes from his correspondence during the period when he began writing the paper, "epistemological considerations were far from Heisenberg's mind during his first attempts to tackle the problem."44

Without necessarily accepting all of Beller's negative judgements about Heisenberg's contributions to the interpretation of quantum mechanics, we can take up her idea that the endorsement of philosophical ideas in his philosophical writings may sometimes be "local and opportunistic" and apply them to the reading of his remarks on the nature of quantum states and quantum probabilities, which Beller does not seem to consider explicitly. From the perspective of her account, it seems natural to read Heisenberg's apparent endorsement of the epistemic conception of states in conjunction with his claims about quantum probabilities as "objective tendencies" mainly as a rhetorical move that should not be mistaken for a serious philosophical account. According to this perspective, the fact that he seems to adopt both the epistemic conception of states and the propensity interpretation of quantum probabilities without demonstrating how these conflicting views may be reconciled is seen as an indication that his primary aim is to be able to avail himself of arguments against his critics that are based on either of them. The dialectical advantages he obtains through this move are the following:

An important criticism of quantum mechanics and its standard interpretation, brought forward, for instance, by Schrödinger ${ }^{45}$, concerns the notion of a "quantum jump", which is central to the "orthodox view" defended by Heisenberg. Historically, the notion of a quantum

40 See (Beller 1999) p. 196

41 See (Beller 1999) p. 199.

42 See (Beller 1999) p. 52.

43 See (Heisenberg 1925), for an English translation see (Heisenberg 1967a).

44 See (Beller 1999) pp. 53-54.

45 See (Schrödinger 1952). 
jump emerged as that of an unruly transition between two energy levels, and it survived in von Neumann's axiomatic codification of the theory in form of wave function collapse that occurs whenever the system is measured. The epistemic conception of states provides Heisenberg with a forceful defence of collapse, which we have encountered many times in the present paper: The sudden change of the quantum state in wave function collapse is not seen as a real physical processes but merely reflects a change in the knowledge of the observer assigning the state to the system. Assuming that the state represents features of the observer's epistemic situation, collapse becomes natural and innocuous. Heisenberg can answer Schrödinger's objection by claiming that "[w]hen the old adage 'Natura non facit saltus' is used as a criticism of quantum theory, we can reply that certainly our knowledge can change suddenly and that this fact satisfies the use of the term 'quantum jump'.".46

Although this reply against critics is certainly very useful for Heisenberg to defend the standard account of the measurement process including collapse, it is not without risks in that by declaring the state subjected to collapse as reflecting subjective information it threatens to invite the charge of being "subjectivist", "instrumentalist", "operationalist", or whatever pejoratively laden form of what today is called "anti-realism" one might think of. From the perspective of the "opportunistic" reading of Heisenberg presented in this section, he declares adherence to an account of quantum probabilities as "objective tendencies" exactly for the purpose of defending himself against this type of charge. The clue of this move is twofold: On the one hand, by presenting an account of quantum probabilities as objective tendencies Heisenberg can point to an at least seemingly clear-cut and precise sense in which quantum mechanics $i s$ objective against those accusing him of subjectivism. On the other hand, this move makes it possible for him to start criticising his own critics by suggesting that their objections arises from a naïve, perhaps nostalgic, desire for classical determinism ${ }^{47}$, which prevents them from recognising the alleged real locus of objectivity in quantum mechanics in the objectivity of quantum mechanical probabilities. By combining an interpretation of quantum probabilities as "objective tendencies" with the epistemic conception of states Heisenberg manages to turn the dialectical situation in the dispute between him and his critics in favour of himself. His opponents may be busy at this stage with rejecting the challenge of naïvely presupposing a deterministic classical world view and therefore may fail to detect the inconsistency (according to this reading) of the package of views which Heisenberg offers.

46 See (Heisenberg 1958) p. 28.

47 This is evident, for instance, in how Heisenberg deals with alternative interpretations of quantum mechanics, portraying them in a very unfavourable light, see Chapter VIII of (Heisenberg 1958). 
Thus, according to this reading, Heisenberg adopts the epistemic conception of states together with an account of quantum probabilities as objective tendencies mainly for rhetorical purposes, not because he is able to combine them in a consistent and unified view. This is certainly not a flattering reading, but it clearly has some advantages and does not seem easy to refute.

\section{Concluding Remark}

It seems difficult to decide which of the readings of Heisenberg's remarks on quantum states developed in the previous sections does best justice to his remarks on quantum states. The reading proposed in the last section, inspired by Beller's view of Heisenberg as a philosophical opportunist, goes perhaps best with the formulations he chooses, and an attractive option might be to accept it without necessarily agreeing with the dismissive overtones of Beller's remarks on Heisenberg as a philosopher of physics. In view of the fact that the most difficult and paradoxical issues in the foundations of quantum mechanics are widely regarded as unresolved as of today, Heisenberg's failure (according to this reading) to deliver a coherent account may be seen as a harmless and easily excusable shortcoming, which pales in comparison to the variety and richness of his ideas on foundational issues. More recent accounts such as those developed by Stapp, Mermin, Fuchs, Healey and others, mentioned in the previous sections, may be seen as modern attempts to combine crucial insights expressed in Heisenberg's writings into more coherent accounts of the foundations of quantum mechanics. My (rather modest) aim in this paper has been to clarify which readings of Heisenberg's remarks on quantum states can be given, which are their most important problems, and which solutions to them are coherent. Arguably, answering these questions is no less a legitimate goal than to determine what Heisenberg's actual view of quantum states really was.

\section{Acknowledgements}

I am very grateful to Koray Karaca and Gregor Schiemann for very helpful comments on an earlier version. Furthermore, I would like to thank two anonymous referees for their useful reports and suggestions on how to improve the paper. 


\section{References}

Beller, Mara. (1999), Quantum Dialogue: The Making of a Revolution, University of Chicago Press, Chicago.

Bub, Jeffrey (2007), Quantum probabilities as degrees of belief, Studies in History and Philosophy of Modern Physics 38(2), pp. 232-254.

Camilleri, Kristian (2009), Heisenberg and the Interpretation of Quantum Mechanics: The Physicist as a Philosopher, Cambridge University Press, Cambridge.

Caves, Carlton M., Fuchs, Christopher A., and Schack, Rüdiger (2002), Quantum probabilities as Bayesian probabilities, Physical Review A 65, p. 022305.

Caves, Carlton M., Fuchs, Christopher A., and Schack, Rüdiger (2007), Subjective probability and quantum certainty, Studies in History and Philosophy of Modern Physics 38(2), pp. 255-274.

d'Espagnat, Bernard (1976), Conceptual Foundations of Quantum Mechanics, $2^{\text {nd }}$ edition, AddisonWesley, Reading, Mass..

Friederich, Simon (2011), How to spell out the epistemic conception of quantum states, Studies in History and Philosophy of Modern Physics 42 (3), pp. 149-157.

Fuchs, Christopher A. (2002), Quantum mechanics as quantum information (and only a little more), in: Khrennikov, A., ed., Quantum Theory: Reconsideration of Foundations Växjö University Press. arXiv:quant-ph/0205039.

Fuchs, Christopher A. and Peres, Asher (2000), Quantum theory needs no 'interpretation', Physics Today 53(3), pp. 70-71.

Fuchs, Christopher A. and Schack, Rüdiger (2010), A quantum-Bayesian route to quantum-state space, Foundations of Physics 41(3), pp. 345-356.

Gillies, Donald (2000), Varieties of Propensity, British Journal for the Philosophy of Science 51, pp. 807-835.

Healey, Richard (2012), Quantum theory: a pragmatist approach, forthcoming in British Journal for 
the Philosophy of Science, doi: 10.1093/bjps/axr054.

Heisenberg, Werner (1925), Über quantentheoretische Umdeutung kinematischer und mechanischer Beziehungen, Zeitschrift für Physik, 33, pp. 841-860, transl. as (Heisenberg 1967a).

Heisenberg, Werner (1934), Wandlungen in den Grundlagen der exacten Naturwissenschaft in jüngster Zeit, Naturwissenschaften, 40, transl. as: Recent changes in the foundations of the exact sciences in (Heisenberg 1979), pp. 11-26. Page references are to (Heisenberg 1979).

Heisenberg, Werner (1955), The development of the interpretation of quantum theory, In: Niels Bohr and the development of physics. Essays dedicated to Niels Bohr on the occasion of his seventieth birthday, ed. by Wolfgang Pauli, Leon Rosenfeld and Victor Weisskopf, McGraw Hill, New York, pp. $12-29$.

Heisenberg, Werner (1958), Physics and Philosophy: The Revolution in Modern Science, George Allen \& Unwin, London, repr. 2007 by Harper, New York, page numbers referring to this edition.

Heisenberg, Werner (1967a) Quantum-theoretical re-interpretation of kinematic and mechanical relations, In: Sources of Quantum Mechanics, ed. B. L. van der Waerden, North-Holland, Amsterdam, pp. 261-276.

Heisenberg, Werner (1971), Physics and beyond, Harper \& Row, NewYork.

Heisenberg, Werner (1979), Philosophical Problems of Quantum Physics, Ox Bow, Woodbridge Conn. [Reprint of Philosophical Problems of Nuclear Science, Pantheon, New York, 1952].

Heisenberg, Werner (1984a), Gesammelte Werke, Series C: Philosophical and popular writings, vol. I: Physik und Erkenntnis 1927-1955, ed. by W. Blum, H. Dürr and H. Rechenberg, Piper, Munich.

Heisenberg, Werner (1984b), Gesammelte Werke, Series C: Philosophical and popular writings, vol. II: Physik und Erkenntnis 1956-1968, ed. by W. Blum, H. Dürr and H. Rechenberg, Piper, Munich.

Marchildon, Louis (2004), Why should we interpret quantum mechanics?, Foundations of Physics 34(10), pp. 1453-1466.

Mermin, N. David (2003), Copenhagen computation, Studies in History and Philosophy of Modern Physics 34(3), pp. 511-522. 
Peierls, Rudolf (1991), In defence of 'Measurement', Physics World January 1991, pp. 19-20.

Pitowsky, Itamar (2003), Betting on the outcomes of measurements: A Bayesian theory of quantum probability, Studies in History and Philosophy of Modern Physics 34(3), pp. 395-414.

Ruetsche, Laura (2002), Interpreting quantum theories, In: The Blackwell Guide to the Philosophy of Science, ed. by Machamer, P. and Silberstein, M., Blackwell, Oxford, pp. 199-226.

Schiemann, Gregor (2008), Werner Heisenberg, C. H. Beck, München.

Schrödinger, Erwin (1952), Are There Quantum Jumps? Part I, The British Journal for the Philosophy of Science 3, pp. 109-123.

Shimony, Abner (1983), Reflections on the philosophy of Bohr, Heisenberg and Schrödinger, in: Physics, Philosophy and Psychoanalysis, ed. by R. S. Cohen and L. Laudan, D. Riedel, Dordrecht, pp. 209-221.

Spekkens, Robert W. (2007), Evidence for the epistemic view of quantum states: A toy theory, Physical Review A 75, p. 032110.

Stapp, Henry P. (2004), Mind, matter and quantum mechanics, $2^{\text {nd }}$ edition, Springer, Berlin, Heidelberg, New York. 